\title{
Granulocyte Colony-Stimulating Factor-Primed Donor Lymphocyte Infusion after Salvage Chemotherapy for Treatment of Relapsed Acute Leukemia after Allogeneic Stem Cell Transplantation
}

\author{
Takeshi Kobayashi, ${ }^{\mathrm{a}}$ Kazuteru Ohashi, ${ }^{\mathrm{a}}$ Miwa Sakai, ${ }^{\mathrm{a}}$ Takuya Yamashita, ${ }^{\mathrm{a}}$ Yoshiki Okuyama, \\ Kiyoshi Hiruma, ${ }^{\mathrm{b}}$ Hideki Akiyama, ${ }^{\mathrm{a}}$ Hisashi Sakamaki ${ }^{\mathrm{a}}$ \\ ${ }^{a}$ Hematology Division and ${ }^{b}$ Blood Transfusion Service, Tokyo Metropolitan Komagome Hospital, Tokyo, Japan
}

Received March 22, 2005; received in revised form April 5, 2005; accepted April 8, 2005

Int J Hematol. 2005;82:79-81. doi: 10.1532/IJH97.05039

(C2005 The Japanese Society of Hematology

\section{Introduction}

The management of posttransplantation relapse is extremely difficult. Although standard reinduction chemotherapy may induce complete remission, long-term diseasefree survival is unlikely because of high treatment-related toxicity and disease recurrence $[1,2]$. Moreover, if reestablishment of donor hematopoiesis is not achieved by reinduction chemotherapy, the profound marrow aplasia that will ensue may result in catastrophic consequences [3]. Here, we describe 3 patients with relapsed acute leukemia after allogeneic bone marrow transplantation (BMT) who developed profound marrow aplasia after aggressive reinduction chemotherapy and who subsequently received granulocyte colony-stimulating factor (G-CSF)-primed donor lymphocyte infusion (DLI) containing abundant $\mathrm{CD} 34^{+}$cells. This strategy was applied in an attempt to reestablish donor hematopoiesis as well as to induce a maximal graft-versusleukemia effect. Although recent reports have demonstrated the plausible efficacy of this approach, none of our 3 patients showed a satisfactory response.

\section{Case Reports}

\subsection{Case 1}

A 34-year-old man with acute lymphoblastic leukemia (ALL) carrying the Philadelphia chromosome with minor $\mathrm{BCR} / \mathrm{ABL}$ transcripts received an allogeneic BMT from his HLA-identical sister during his first remission in October

Correspondence and reprint requests: Kazuteru Ohashi, MD, FACP, Hematology Division, Tokyo Metropolitan Komagome Hospital, 3-18-22 Honkomagome Bunkyo-ku, Tokyo 113-8677, Japan; 81-3-3823-2101; fax: 81-3-3824-1552 (e-mail: k.ohashi@ cick.jp).
1999. The initial posttransplantation course was unremarkable; however, the patient relapsed at day 152 . The patient was then given intensive salvage chemotherapy using a doseadjusted RELAL-88 5-day induction regimen (vindesine, mitoxantrone, cyclophosphamide, intermediate-dose cytarabine, prednisolone, and methotrexate) [4]. After completion of this chemotherapy regimen, the patient achieved a hematologic remission. The patient was then treated with DLI from the original donor, as previously described [5]. Despite these aggressive interventions, which resulted in temporary molecular remission [5], the patient again relapsed at day 561. The patient received the same therapeutic strategy applied at the initial relapse. However, the aggressive chemotherapy performed on day 578 resulted in profound myelosuppression; therefore, at day 605 the patient received G-CSF-primed DLI rather than unprimed DLI. No further chemotherapy and no graft-versus-host disease (GVHD) prophylaxis were administered before the G-CSF-primed DLI. Although the patient developed grade II acute GVHD affecting the gut and liver, treatment with prednisolone and FK506 stabilized the patient well. The hematologic recovery was rapid and observed as early as 11 days after the infusion of G-CSF-primed DLI, but this strategy failed to induce a complete hematologic response, as indicated by the small number of blasts still detected in a bone marrow aspirate obtained on day 616. The minimal residual disease state assessed by real-time reverse transcriptase-polymerase chain reaction analysis of minor $\mathrm{BCR} / \mathrm{ABL}$ messenger RNA performed before and after treatment was 650,000 and 1600 copies/ $\mu \mathrm{g}$ transfer RNA, respectively. Six months after G-CSF-primed DLI, the patient died of relapse after an abrupt increase in peripheral blood leukemic blasts.

\subsection{Case 2}

A 27-year-old woman with Philadelphia chromosomenegative ALL underwent an allogeneic BMT from her HLAidentical brother during the early relapse phase in December 

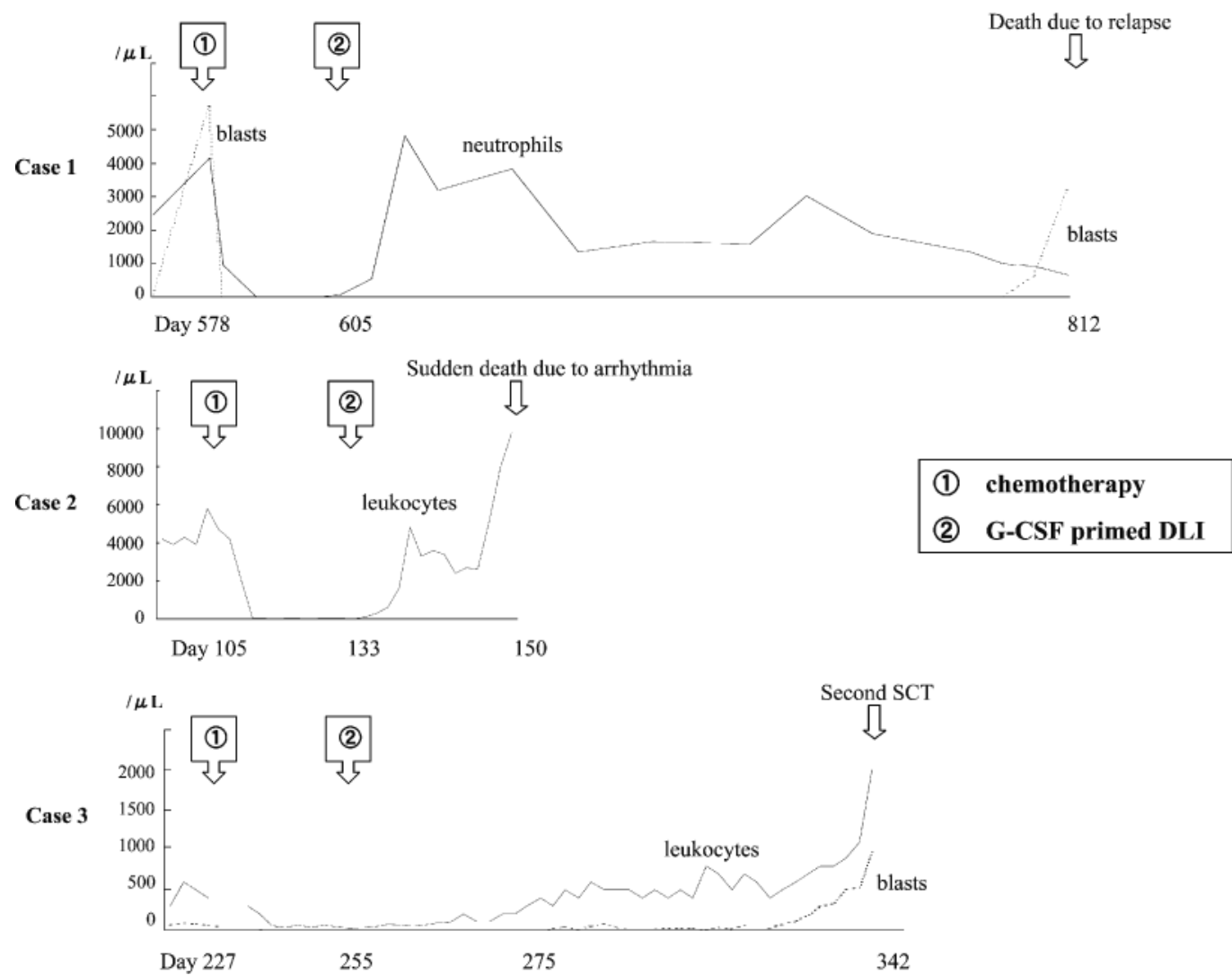

Figure 1. Clinical courses of the 3 patients. The serial changes in the numbers of neutrophils (solid lines) and blasts (dashed lines) are shown. Granulocyte colony-stimulating factor (G-CSF)-primed donor lymphocyte infusions (DLI) contained $1.47 \times 10^{6} / \mathrm{kg} \mathrm{CD}^{+} 4^{+}$cells and $2.34 \times 10^{8} / \mathrm{kg} \mathrm{CD} 3^{+}$ cells (case 1), $1.15 \times 10^{6} / \mathrm{kg} \mathrm{CD} 34^{+}$cells and $2.78 \times 10^{8} / \mathrm{kg} \mathrm{CD}^{+}$cells (case 2), and $1.76 \times 10^{6} / \mathrm{kg} \mathrm{CD} 34^{+}$cells and $3.58 \times 10^{8} / \mathrm{kg} \mathrm{CD} 3^{+}$cells $($case 3 ). SCT indicates stem cell transplantation.

2003. However, the patient relapsed as early as 3 months after the initial transplantation. Abrupt discontinuation of cyclosporine resulted in no response. Therefore, the patient received the same chemotherapy applied in case 1 on day 105, but she developed severe aplasia. Consequently, G-CSF-primed donor lymphocytes were infused on day 133. The patient received no conditioning therapy and no GVHD prophylaxis. Although a relatively low number of CD34+ cells were infused, the patient showed rapid hematologic recovery and became transfusion independent, as is shown in Figure 1. The patient also developed grade II acute GVHD of the skin, which was treated with methylprednisolone $(1 \mathrm{mg} / \mathrm{kg}$ per day). Improvement in the skin GVHD permitted tapering of the dosage. However, 17 days after G-CSF-primed DLI, the patient suddenly died of treatmentrelated arrhythmia. An autopsy revealed no evidence of ALL recurrence.

\subsection{Case 3}

A 23-year-old man with acute myelogenous leukemia underwent an allogeneic BMT from an HLA-identical brother during his first remission in September 2003 but relapsed 5 months later. The patient received standard reinduction chemotherapy consisting of cytarabine and idarubicin but did not achieve a hematologic remission. Therefore, the patient was put on further aggressive chemotherapy with the time-sequential application of mitoxantrone plus intermediate-dose cytarabine followed by high-dose cytarabine plus etoposide on day 227 [6]. However, the patient developed severe aplasia after chemotherapy; consequently, G-CSF-primed lymphocytes were infused on day 255 . This attempt appeared to induce no hematologic response, as determined by the existence of $60 \%$ leukemic blasts in the patient's bone marrow sample obtained on day 276 . However, the patient was followed up and was in good general condition without an abrupt increase in peripheral leukemic blasts until day 342 , when a second transplantation was scheduled. This patient also did not receive any GVHD prophylaxis, but he did not develop acute or chronic GVHD.

\section{Discussion}

Several reports have indicated that the combination of chemotherapy and DLI provides little impact on overall survival in patients with relapsed acute leukemia [7]. Recently, Glass et al reported the use of G-CSF-primed DLI, instead of unprimed DLI, after chemotherapy for treatment of patients with relapsed leukemia [8]. These authors' intention was to circumvent some of the problems associated with 
unprimed DLI. First, the infusion of a high number of donor CD $34^{+}$cells might avoid aplasia after DLI. Second, the transfer of a complete progenitor cell graft might allow the wider use of aggressive chemotherapy prior to DLI. Although this approach is very close to that taken in a second transplantation, the crucial ways in which it differs from a second transplantation are the use of nonmyeloablative chemotherapy and the absence of prophylactic immunosuppression for acute GVHD. The available data from 2 prospective studies have disclosed a high rate of complete remission with this strategy. Levine et al reported a maximal response rate of 59\% in selected patients [9]. Another study by Choi et al has also shown promising results: the complete remission rate and the 2-year overall survival rate were $63 \%$ and $31 \%$, respectively [10]. These results were surprisingly superior to the historical data for DLI (complete remission rates of $0 \%-29 \%$ ) [11]. Our approach was essentially the same as that of these studies, including the use of GCSF-primed DLI and the absence of GVHD prophylaxis, although our patients received DLI with a relatively long interval (28-29 days in our study versus 7-15 days in the previous 2 studies). We performed G-CSF-primed DLI as stem cell rescue rather than as front-line therapy. However, in contrast to the previous 2 reports, our experience of these 3 patients provided only dismal outcomes, with 1 sudden death associated with treatment-related toxicity. Although our small study population, including advanced leukemia with short remission duration after initial BMT, may prohibit drawing meaningful conclusions, these data will be the basis for further studies with larger numbers of patients to assess this new treatment strategy.

\section{References}

1. Giralt SA, Champlin RE. Leukemia relapse after allogeneic bone marrow transplantation: a review. Blood. 1994;84:3603-3612.
2. Frassoni F, Barrett AJ, Granena A, et al. Relapse after allogeneic bone marrow transplantation for acute leukemia: a survey by the EBMT of 117 cases. Br J Haematol. 1988;70:317-320.

3. Keil F, Prinz E, Kalhs P, et al. Treatment of leukemic relapse after allogeneic stem cell transplantation with cytoreductive chemotherapy and/or immunotherapy or second transplants. Leukemia. 2001;15:355-361.

4. Martino R, Bellido M, Brunet S, et al. Intensive salvage chemotherapy for primary refractory or first relapsed adult acute lymphoblastic leukemia: results of a prospective trial. Haematologica. 1999;84:505-510.

5. Kono N, Ohashi K, Okuyama Y, et al. Treatment of relapsing $\mathrm{Ph}^{+}$acute lymphoblastic leukemia with donor leukocyte infusion followed by quantitative monitoring of residual disease. Hematology. 2001;6:261-265.

6. Martino R, Guardia R, Altes A, et al. Time sequential chemotherapy for primary refractory or relapsed adult acute myeloid leukemia: results of the phase II GEMIA protocol. Haematologica. 1999;84:226-230.

7. Collins RH Jr, Goldstein S, Giralt S, et al. Donor leukocyte infusions in acute lymphocytic leukemia. Bone Marrow Transplant. 2000;26:511-516.

8. Glass B, Majolino I, Dreger P, et al. Allogeneic peripheral blood progenitor cells for treatment of relapse after bone marrow transplantation. Bone Marrow Transplant. 1997;20:533-541.

9. Levine JE, Braun T, Penza SL, et al. Prospective trial of chemotherapy and donor leukocyte infusions for relapse of advanced myeloid malignancies after allogeneic stem-cell transplantation. J Clin Oncol. 2002;20:405-412.

10. Choi SJ, Lee JH, Kim S, et al. Treatment of relapsed acute myeloid leukemia after allogeneic bone marrow transplantation with chemotherapy followed by G-CSF-primed donor leukocyte infusion: a high incidence of isolated extramedullary relapse. Leukemia. 2004;18:1789-1797.

11. Kolb HJ, Schattenberg A, Goldman JM, et al. Graft-versusleukemia effect of donor lymphocyte transfusions in marrow grafted patients: European Group for Blood and Marrow Transplantation Working Party Chronic Leukemia. Blood. 1995;86: 2041-2050. 